\title{
Deformation Quantization in the Teaching of Quantum Mechanics
}

\author{
Allen C. Hirshfeld* and Peter Henselder ${ }^{\dagger}$ \\ Fachbereich Physik \\ Universität Dortmund \\ 44221 Dortmund
}

October 22, 2018

\begin{abstract}
We discuss the deformation quantization approach for the teaching of quantum mechanics. This approach has certain conceptual advantages that make its consideration worthwhile. In particular, it sheds new light on the relation between classical and quantum mechanics. We demonstrate how it can be used to solve specific problems and clarify its relation to conventional quantization and path integral techniques. We also discuss its recent applications in relativistic quantum field theory.
\end{abstract}

\section{Introduction}

In this article we discuss and compare three approaches to quantum mechanics: the operator formalism, the path integral approach, and deformation quantization. Conventional texts use the formalism in which the observables are represented by operators in Hilbert space, an approach that goes back to Diradt and von Neumann. 3 The path integral approach was initiated by Feynman and is widely used today in research in quantum field theory. For this reason it is also discussed in some introductory treatments, for example, in Ref. [4], and in more advanced texts. 0 . Some of the techniques used in deformation quaptization were introduced by the pioneers of quantum mechanics (Wigner, Weyl, von Neumanne), but it was first proposed as an autonomous theory by Bayen et al. 1 in 1978. Since then, many other articles have been written on the topic. For recent reviews see Refs. [9]-12].

Deformation quantization concentrates on the central physical concepts of quantum theory: the algebra of observables and their dynamical evolution. 13 Because it deals exclusively with functions of phase space variables, its conceptual break with classical mechanics is less severe than in other approaches. It gives the correspondence principle, which played such an important role in the historical development, a precise formulation. It is set in the framework of Poisson manifolds, which are an important generalization of the usual symplectic case, and which are needed for the formulation of gauge field theories. Many of its results can be rigorously established using adaptions of known techniques or completely new approaches. For these reasons it may well lead to progress in treating problems in quantum field theory 14 as well as in ordinary quantum mechanics, where its techniques already enjoy a measure of popularity, see for example the references in 12 . Recently it has received an important impetus from new developments in mathematics, 15 recognized by Kontsevich's Fields medal in 1998. Some believe it will supplant, or at least complement,

\footnotetext{
*hirsh@physik.uni-dortmund.de

†henselde@dilbert.physik.uni-dortmund.de
} 
the other methods in quantum mechanics and quantum field theory. As an autonomous approach to quantum theory, its conceptual advantages recommend its use in graduate instruction.

In this article we sketch how one might go about teaching quantum mechanics according to this approach. Background material at the appropriate leyel can be found in the texts by Marsden and Ratiu, 16 Vaisman 17 and da Silva and Weinstein.18 In Sec. 2 we briefly review the fundamental concepts of the Hamiltonian formalism for classical mechanics and their generalization to the setting of Poisson manifolds. In Sec. 3 we introduce the main tool of deformation quantization, the star product, which deforms the commutative classical algebra of observables into the non-commutative quantum algebra of observables. Having chosen a quantization scheme one can proceed to calculate the physical quantities of interest for a given system, following the method presented in Sec. 4. These techniques are illustrated in Sec. 5 for the case of the simple harmonic oscillator. Section 6 gives a short review of conventional quantization, in preparation for Sec. 7, which compares the conventional approach to deformation quantization and to path integral methods. Finally, Sec. 8 gives an overview of some important applications of deformation quantization in relativistic quantum field theory. The appendix demonstrates calculational techniques useful in this context, and suggests some exercises that students can do to familiarize themselves with the material.

\section{Classical Mechanics and Poisson Manifolds}

Quantum mechanics and the canonical Hamiltonian formalism for classical mechanics have always been closely related. When we speak of a classical dynamical system with a finite number of degrees of freedom, we have in mind something like an $n$-particle system, where the particles are specified at any time by their instantaneous positions and momenta. That is, the state of the system is specified as a point in the $2 n$-dimensional phase space $M . M$ is a smooth manifold, and in canonical coordinates a point $x$ in $M$ is written as $x=(q, p)=\left(q_{1}, \ldots, q_{n}, p_{1}, \ldots, p_{n}\right)$.

The observables of the system, such as the Hamilton function for example, are smooth realvalued functions on this phase space. Physical quantities of the system at some time, such as the energy, are calculated by evaluating the Hamilton function at the point in phase space $x_{0}=\left(q_{0}, p_{0}\right)$ that characterizes the state of the system at this time. The mathematical expression for this operation is

$$
E=\int H(q, p) \delta^{(2)}\left(q-q_{0}, p-p_{0}\right) d q d p,
$$

where $\delta^{(2)}$ is the two-dimensional Dirac delta function (we assume for notational simplicity a oneparticle system). The observables of the dynamical system are functions on the phase space, the states of the system are positive functionals on the observables (here the Dirac delta functions), and we obtain the value of the observable in a definite state by the operation shown in Eq. (2.1).

In general, functions on a manifold are multiplied by each other in a pointwise manner, that is, given two functions $f$ and $g$, their product $f g$ is the function

$$
(f g)(x)=f(x) g(x) .
$$

In the context of classical mechanics we say that the observables build a commutative algebra, and we speak of the commutative classical algebra of observables.

In Hamiltonian mechanics there is another way to combine two functions on phase space in such a way that a further function on phase space results, namely by use of the Poisson bracket

$$
\{f, g\}(q, p)=\left.\sum_{i=1}^{n}\left(\frac{\partial f}{\partial q_{i}} \frac{\partial g}{\partial p_{i}}-\frac{\partial f}{\partial p_{i}} \frac{\partial g}{\partial q_{i}}\right)\right|_{q, p} .
$$


Note that here the new function results not by using the values of the functions $f$ and $g$ at the given point in phase space, but rather by using the values of their derivatives at this point.

Because expressions like those in Eq. (2.3) are used frequently, it is advantageous to employ a compact notation. We provide the derivatives with vector symbols, which indicate if they act on functions to the right or to the left. For example,

$$
f \overleftarrow{\partial}_{q_{i}} g=\frac{\partial f}{\partial q_{i}} g, \quad f \vec{\partial}_{p_{i}} g=f \frac{\partial g}{\partial p_{i}},
$$

and Eq. 2.3) is written as

$$
\{f, g\}=\sum_{i} f\left(\overleftarrow{\partial}_{q_{i}} \vec{\partial}_{p_{i}}-\overleftarrow{\partial}_{p_{i}} \vec{\partial}_{q_{i}}\right) g .
$$

From now on we shall use the Einstein convention of summing over repeated indices. When there is no danger of confusion, we sometimes suppress the indices altogether. In such cases the notation would strictly be correct only for one-particle systems; for many-particle systems the summed indices are left implicit. Eq. (2.3) can then be written as

$$
\{f, g\}=f\left(\overleftarrow{\partial}_{q} \vec{\partial}_{p}-\overleftarrow{\partial}_{p} \vec{\partial}_{q}\right) g .
$$

We may abbreviate our notation further by using $x$ to represent points of the phase space manifold, $x=\left(x_{1}, \ldots, x_{2 n}\right)$, and introducing the Poisson tensor $\alpha^{i j}$, where the indices $i, j$ run from 1 to $2 n$. In canonical coordinates $\alpha^{i j}$ is represented by the matrix

$$
\alpha=\left(\begin{array}{cc}
0 & -I_{n} \\
I_{n} & 0
\end{array}\right),
$$

where $I_{n}$ is the $n \times n$ identity matrix. Then Eq. (2.3) becomes

$$
\{f, g\}(x)=\alpha^{i j} \partial_{i} f(x) \partial_{j} g(x),
$$

where $\partial_{i}=\partial / \partial x_{i}$.

The time development of the system is given by Hamilton's equations, which are easily expressed in terms of the Poisson brackets:

$$
\dot{q}_{i}=\frac{\partial H}{\partial p_{i}}=\left\{q_{i}, H\right\}, \quad \dot{p}_{i}=-\frac{\partial H}{\partial q_{i}}=\left\{p_{i}, H\right\} .
$$

For a general observable

$$
\dot{f}=\{f, H\} .
$$

Because $\alpha$ transforms like a tensor with respect to coordinate transformations, Eq. (2.8) may also be written in noncanonical coordinates. In this case the components of $\alpha$ need not be constants, and may depend on the point of the manifold at which they are evaluated. But in Hamiltonian mechanics, $\alpha$ is still required to be invertible. A manifold equipped with a Poisson tensor of this kind is called a symplectic manifold. In modern treatments of mechanics, such as in Ref. [16], one uses a more general framework. The tensor $\alpha$ is no longer required to be invertible, but it nevertheless suffices to define Poisson brackets via Eq. (2.8), and these brackets are required to have the properties

(i) $\{f, g\}=-\{g, f\}$,

(ii) $\{f, g h\}=\{f, g\} h+g\{f, h\}$, 
(iii) $\{f,\{g, h\}\}+\{g,\{h, f\}\}+\{h,\{f, g\}\}=0$.

Property (i) tells us that the Poisson bracket is antisymmetric, property (ii) is referred to as the Leibnitz rule, and property (iii) is called the Jacobi identity. The Poisson bracket used in Hamiltonian mechanics satisfies all these properties, but we now abstract these properties from the concrete prescription of Eq. (2.3), and define a Poisson manifold $(M, \alpha)$ as a smooth manifold $M$ equipped with a Poisson tensor $\alpha$, whose components are no longer necessarily constant, such that the bracket defined by Eq. (2.8) has the above properties. It turns out that such manifolds provide a better context for treating dynamical systems with symmetries. In fact, they are essential for treating gauge field theories, which govern the fundamental interactions of elementary particles.

\section{Quantum Mechanics and Star Products}

Up to now we have been considering classical mechanics. The essential difference between classical and quantum mechanics is Heisenberg's uncertainty relation, which implies that in the latter, states can no longer be represented as points in phase space. The uncertainty is a consequence of the non-commutativity of the quantum mechanical observables. That is, the commutative classical algebra of observables must be replaced by a non-commutative quantum algebra of observables.

In the conventional approach to quantum mechanics this non-commutativity is implemented by representing the quantum mechanical observables by linear operators in Hilbert space. Physical quantities are then represented by eigenvalues of these operators, and physical states are related to the operator eigenfunctions. Although these entities are somehow related to their classical counterparts, to which they are supposed to reduce in an appropriate limit, the precise relationship has remained obscure, one hundred years after the beginnings of quantum mechanics. Textbooks refer to the correspondence principle, which guided the pioneers of the subject. Attempts to give this idea a precise formulation by postulating a specific relation between the classical Poisson brackets of observables and the commutators of the corresponding quantum mechanical operators, as undertaken for example by Dirac and von Neumann, encounter insurmountable difficulties, as pointed out by Groenewold in 1946 in an unjustly neglected paper 19 In the same paper Groenewold also wrote down the first explicit representation of a star product (see Eq. (3.5)), without however realizing the potential of this concept for overcoming the difficulties that he wanted to resolve.

In the deformation quantization approach there is no such break when going from the classical system to the corresponding quantum system; we describe the quantum system by using the same entities that we use to describe the classical system. The observables of the system are described by the same functions on phase space as their classical counterparts. Uncertainty is realized by describing physical states as distributions on phase space that are not sharply localized, in contrast to the Dirac delta functions which occur in the classical case. When we evaluate an observable in some definite state according to the quantum analogue of Eq. (2.1) (see Eq. (4.5)), values of the observable in a whole region contribute to the number that we obtain, which is thus an average value of the observable in the given state. Non-commutativity is incorporated by introducing a non-commutative product for functions on phase space, so that we get a new non-commutative quantum algebra of observables.

The passage from the classical algebra of observables to the quantum algebra of observables is performed in a continuous fashion. When mathematicians investigate a particular structure, they try to modify it in various ways in order to see how these modifications affect it: which properties are preserved under certain modifications and which properties change. If the modification changes the structure in a continuous fashion, they speak of a deformation. All work on deformation quantization stems from Gerstenhaber's seminal 1964 paper,20 where he introduced the concept of a star product of smooth functions on a manifold.

For applications to quantum mechanics we consider smooth complex-valued functions on a Poisson manifold. A star product $f * g$ of two such functions is a new smooth function, which, in 
general, is described by an infinite power series:

$$
f * g=f g+(i \hbar) C_{1}(f, g)+O\left(\hbar^{2}\right)=\sum_{n=0}^{\infty}(i \hbar)^{n} C_{n}(f, g) .
$$

The first term in the series is the pointwise product given in Eq. (2.2), and $(i \hbar)$ is the deformation parameter, which we think of as varying continuously. If $\hbar$ is identified with Planck's constant, then what varies is really the magnitude of the action of the dynamical system considered in units of $\hbar$ : the classical limit holds for systems with large action. In this limit, which we express here as $\hbar \rightarrow 0$, the star product reduces to the usual product. In general, the coefficients $C_{n}$ will be such that the new product is non-commutative, and we speak of the non-commutative algebra formed from the functions with this new multiplication law as a deformation of the original commutative algebra, which uses pointwise multiplication of the functions.

The expressions $C_{n}(f, g)$ denote functions made up of the derivatives of the functions $f$ and $g$. Examples will be given below, as in Eq. (3.4). It is obvious that without further restrictions of these coefficients, the star product is too arbitrary to be of any use. Gerstenhaber's discovery was that the simple requirement that the new product be associative imposes such strong requirements on the coefficients $C_{n}$ that they are essentially unique in the most important cases (up to an equivalence relation which we shall discuss below). Formally, Gerstenhaber required that the coefficients satisfy the following properties:

(1) $\sum_{j+k=n} C_{j}\left(C_{k}(f, g), h\right)=\sum_{j+k=n} C_{j}\left(f, C_{k}(g, h)\right)$

(2) $C_{0}(f, g)=f g$

(3) $C_{1}(f, g)-C_{1}(g, f)=\{f, g\}$.

Property (1) guarantees that the star product is associative: $(f * g) * h=f *(g * h)$. Property (2) means that in the limit $\hbar \rightarrow 0$ the star product $f * g$ agrees with the pointwise product $f g$. Property (3) has at least two aspects. Mathematically, it anchors the new product to the given structure of the Poisson manifold. Physically, it provides the connection between the classical and quantum behavior of the dynamical system. Define a commutator by using the new product:

$$
[f, g]_{*}=f * g-g * f .
$$

Property (3) may then be written as

$$
\lim _{\hbar \rightarrow 0} \frac{1}{i \hbar}[f, g]_{*}=\{f, g\} .
$$

Equation (3.3) is the correct form of the correspondence principle. In general, the quantity on the left-hand side of Eq. (3.3) reduces to the Poisson bracket only in the classical limit. The source of the mathematical difficulties that previous attempts to formulate the correspondence principle encountered was related to trying to enforce equality between the Poisson bracket and the corresponding expression involving the quantum mechanical commutator. Equation (3.3) shows that such a relation in general only holds up to corrections of higher order in $\hbar$.

For physical applications we usually require the star product to be Hermitean: $\overline{f * g}=\bar{g} * \bar{f}$, where $\bar{f}$ denotes the complex conjugate of $f$. The star products we are mainly concerned with in the following have this property.

For a given Poisson manifold it is not clear a priori if a star product for the smooth functions on the manifold actually exists, that is, whether it is at all possible to find coefficients $C_{n}$ that satisfy the above list of properties. Even if we find such coefficients, it it still not clear that the series they define through Eq. (3.1) yields a smooth function. Mathematicians have worked hard 
to answer these questions in the general case.21 For flat Euclidian spaces, $M=\mathbb{R}^{2 n}$, a specific star product has long been known. In this case the components of the Poisson tensor $\alpha^{i j}$ can be taken to be constants. The coefficient $C_{1}$ can then be chosen antisymmetric, so that

$$
C_{1}(f, g)=\frac{1}{2} \alpha^{i j}\left(\partial_{i} f\right)\left(\partial_{j} g\right)=\frac{1}{2}\{f, g\}
$$

by property (3) above. The higher order coefficients may be obtained by exponentiation of $C_{1}$. This procedure yields the Moyal star product: 19.22

$$
f *_{M} g=f e^{\left(\frac{i \hbar}{2}\right) \alpha^{i j} \overleftarrow{\partial}_{i} \vec{\partial}_{j}} g
$$

In canonical coordinates Eq. (3.5) becomes

$$
\begin{aligned}
\left(f *_{M} g\right)(q, p) & =f(q, p) e^{\frac{i \hbar}{2}\left(\overleftarrow{\partial}_{q} \vec{\partial}_{p}-\overleftarrow{\partial}_{p} \vec{\partial}_{q}\right)} g(q, p) \\
& =\sum_{m, n=0}^{\infty}\left(\frac{i \hbar}{2}\right)^{m+n} \frac{(-1)^{m}}{m ! n !}\left(\partial_{p}^{m} \partial_{q}^{n} f\right)\left(\partial_{p}^{n} \partial_{q}^{m} g\right)
\end{aligned}
$$

We now come to the question of uniqueness of the star product on a given Poisson manifold. Two star products $*$ and $*^{\prime}$ are said to be c-equivalent if there exists an invertible transition operator

$$
T=1+\hbar T_{1}+\cdots=\sum_{n=0}^{\infty} \hbar^{n} T_{n}
$$

where the $T_{n}$ are differential operators, that satisfies

$$
f *^{\prime} g=T^{-1}((T f) *(T g))
$$

It is known that for $M=\mathbb{R}^{2 n}$ all admissible star products are $c$-equivalent to the Moyal product. An example of another star product in $\mathbb{R}^{2 n}$ is the standard star product, defined by

$$
f *_{S} g=f e^{i \hbar \overleftarrow{\partial}_{q} \vec{\partial}_{p}} g
$$

The Moyal and standard star products are $c$-equivalent, that is,

$$
T\left(f *_{S} g\right)=(T f) *_{M}(T g),
$$

with the transition operator

$$
T=e^{-\frac{i \hbar}{2} \vec{\partial}_{q} \vec{\partial}_{p}}
$$

Note that the antisymmetric part of the differential operator in the exponent of the standard product in Eq. (3.10) equals that of the exponent of the Moyal product in Eq. (3.6). This equality is a general feature of $c$-equivalent star products: it follows from condition (3) for the star product, which both forms have to obey. For more general manifolds the equivalence question has been studied in Ref. [23]. The concept of $c$-equivalence is a mathematical one $(c$ stands for cohomology 20 ); it does not by itself imply any kind of physical equivalence, as we shall see below.

Before concluding this section we present two alternative expressions for the Moyal star product. A form that often is useful in calculations is given by the shift formula

$$
\left(f *_{M} g\right)(q, p)=f\left(q+\frac{i \hbar}{2} \vec{\partial}_{p}, p-\frac{i \hbar}{2} \vec{\partial}_{q}\right) g(q, p)
$$


which can be obtained from the definition, Eq. (3.7), by repeated applications of the Taylor formula in the form given in Eq. (A.6) in the Appendix Still another expression for the Moyal product, important both in theory and applications, 2425 is a kind of Fourier representation:

$$
\begin{aligned}
\left(f *_{M} g\right)(q, p) & =\frac{1}{\hbar^{2} \pi^{2}} \int d q_{1} d q_{2} d p_{1} d p_{2} f\left(q_{1}, p_{1}\right) g\left(q_{2}, p_{2}\right) \\
& \times \exp \left[\frac{2}{i \hbar}\left(p\left(q_{1}-q_{2}\right)+q\left(p_{2}-p_{1}\right)+\left(q_{2} p_{1}-q_{1} p_{2}\right)\right] .\right.
\end{aligned}
$$

A derivation of this expression is given in the Appendix. Equation (3.14) has an interesting geometrical interpretation. 20 Denote points in phase space by vectors, for example in two dimensions

$$
\mathbf{r}=\left(\begin{array}{c}
q \\
p
\end{array}\right), \quad \mathbf{r}_{1}=\left(\begin{array}{c}
q_{1} \\
p_{1}
\end{array}\right), \quad \mathbf{r}_{2}=\left(\begin{array}{c}
q_{2} \\
p_{2}
\end{array}\right)
$$

Now consider the triangle in phase space spanned by the vectors $\mathbf{r}-\mathbf{r}_{1}$, and $\mathbf{r}-\mathbf{r}_{2}$. Its area (symplectic volume) is

$$
\begin{aligned}
A\left(\mathbf{r}, \mathbf{r}_{1}, \mathbf{r}_{2}\right) & =\frac{1}{2}\left(\mathbf{r}-\mathbf{r}_{1}\right) \wedge\left(\mathbf{r}-\mathbf{r}_{2}\right) \\
& =\frac{1}{2}\left[p\left(q_{2}-q_{1}\right)+q\left(p_{1}-p_{2}\right)+\left(q_{1} p_{2}-q_{2} p_{1}\right)\right],
\end{aligned}
$$

which is proportional to the exponent in Eq. (3.14). Hence we may rewrite Eq. (3.14) as

$$
(f * g)(\mathbf{r})=\int d \mathbf{r}_{1} d \mathbf{r}_{2} f\left(\mathbf{r}_{1}\right) g\left(\mathbf{r}_{2}\right) \exp \left[\frac{4 i}{\hbar} A\left(\mathbf{r}, \mathbf{r}_{1}, \mathbf{r}_{2}\right)\right] .
$$

We shall meet this equation again at the end of this article.

\section{Deformation Quantization}

The properties of the star product are well adapted for describing the noncommutative quantum algebra of observables. We have already discussed the associativity and the incorporation of the classical and semi-classical limits. Note that the characteristic non-locality feature of quantum mechanics is also explicit. In the expression for the Moyal product given in Eq. (3.7) the star product of the functions $f$ and $g$ at the point $x$ involves not only the values of the functions $f$ and $g$ at this point, but also all higher derivatives of these functions at $x$. But for a smooth function, knowledge of all the derivatives at a given point is equivalent to knowledge of the function on the entire space. In the integral expression of Eq. (3.14) we also see that knowledge of the functions $f$ and $g$ on the whole phase space is necessary to determine the value of the star product at the point $x=(q, p)$.

The $c$-equivalent star products correspond to different quantization schemes. Having chosen a quantization scheme, the quantities of interest for the quantum system may be calculated. It turns out that different quantization schemes lead to different spectra for the observables. The choice of a specific quantization scheme can only be motivated by further physical requirements. In the simple example we discuss below, the classical system is completely specified by its Hamilton function. In more general cases one may have to decide what constitutes a sufficiently large set of good observables for a complete specification of the system.

A state is characterized by its energy $E$, the set of all possible values for the energy is called the spectrum of the system. The states are described by distributions on phase space called projectors. The state corresponding to the energy $E$ is denoted by $\pi_{E}(q, p)$. These distributions are normalized:

$$
\frac{1}{2 \pi \hbar} \int \pi_{E}(q, p) d q d p=1
$$


and idempotent:

$$
\left(\pi_{E} * \pi_{E^{\prime}}\right)(q, p)=\delta_{E, E^{\prime}} \pi_{E}(q, p) .
$$

The fact that the Hamilton function takes the value $E$ when the system is in the state corresponding to this energy is expressed by the equation

$$
\left(H * \pi_{E}\right)(q, p)=E \pi_{E}(q, p) .
$$

Equation (4.3) corresponds to the time-independent Schrödinger equation, and is sometimes called the $*$-genvalue equation. The spectral decomposition of the Hamilton function is given by

$$
H(q, p)=\sum_{E} E \pi_{E}(q, p)
$$

where the summation sign may indicate an integration if the spectrum is continuous. The quantum mechanical version of Eq. (2.1) is

$$
E=\frac{1}{2 \pi \hbar} \int\left(H * \pi_{E}\right)(q, p) d q d p=\frac{1}{2 \pi \hbar} \int H(q, p) \pi_{E}(q, p) d q d p,
$$

where the last expression may be obtained by using Eq. (3.14) for the star product.

The time-evolution function for a time-independent Hamilton function is denoted by $\operatorname{Exp}(H t)$, and the fact that the Hamilton function is the generator of the time-evolution of the system is expressed by

$$
i \hbar \frac{d}{d t} \operatorname{Exp}(H t)=H * \operatorname{Exp}(H t)
$$

This equation corresponds to the time-dependent Schrödinger equation. It is solved by the star exponential:

$$
\operatorname{Exp}(H t)=\sum_{n=0}^{\infty} \frac{1}{n !}\left(\frac{-i t}{\hbar}\right)^{n}(H *)^{n}
$$

where $(H *)^{n}=\underbrace{H * H * \cdots * H}_{n \text { times }}$. Because each state of definite energy $E$ has a time-evolution $e^{-i E t / \hbar}$, we expect that the complete time-evolution function may be written in the form:

$$
\operatorname{Exp}(H t)=\sum_{E} \pi_{E} e^{-i E t / \hbar}
$$

This expression is called the Fourier-Dirichlet expansion for the time-evolution function.

Questions concerning the existence and uniqueness of the star exponential as a $C^{\infty}$ function and the nature of the spectrum and the projectors again require careful mathematical analysis. The problem of finding general conditions on the Hamilton function $H$ which ensure a reasonable physical spectrum is analogous to the problem of showing in the conventional approach that the symmetric operator $\hat{H}$ is self-adjoint and finding its spectral projections. Some of these questions have been answered by Hansen.24 Others are the subject of ongoing research. But let us not forget that quantum mechanics is a physical theory. Physicists are usually interested in specific systems, and one can often determine empirically if some quantity exists just by calculating it! This will turn out to be the case in the following example.

\section{The Simple Harmonic Oscillator}

The simple one-dimensional harmonic oscillator is characterized by the classical Hamilton function

$$
H(q, p)=\frac{p^{2}}{2 m}+\frac{m \omega^{2}}{2} q^{2} .
$$


In terms of the holomorphic variables

$$
a=\sqrt{\frac{m \omega}{2}}\left(q+i \frac{p}{m \omega}\right), \bar{a}=\sqrt{\frac{m \omega}{2}}\left(q-i \frac{p}{m \omega}\right)
$$

the Hamilton function becomes

$$
H=\omega a \bar{a} .
$$

Our aim is to calculate the time-evolution function. We first choose a quantization scheme characterized by the normal star product

$$
f *_{N} g=f e^{\hbar \bar{\partial}_{a} \vec{\partial}_{\bar{a}}} g .
$$

We then have

$$
\bar{a} *_{N} a=a \bar{a}, a *_{N} \bar{a}=a \bar{a}+\hbar,
$$

so that

$$
[a, \bar{a}]_{*_{N}}=\hbar .
$$

Equation (4.6) for this case is

$$
i \hbar \frac{d}{d t} \operatorname{Exp}_{N}(H t)=\left(H+\hbar \omega \bar{a} \partial_{\bar{a}}\right) \operatorname{Exp}_{N}(H t),
$$

with the solution

$$
\operatorname{Exp}_{N}(H t)=e^{-a \bar{a} / \hbar} \exp \left(e^{-i \omega t} a \bar{a} / \hbar\right) .
$$

By expanding the last exponential in Eq. (5.8), we obtain the Fourier-Dirichlet expansion:

$$
\operatorname{Exp}_{N}(H t)=e^{-a \bar{a} / \hbar} \sum_{n=0}^{\infty} \frac{1}{\hbar^{n} n !} \bar{a}^{n} a^{n} e^{-i n \omega t} .
$$

If we compare coefficients in Eqs. (4.8) and (5.9), we find

$$
\begin{aligned}
\pi_{0}^{(N)} & =e^{-a \bar{a} / \hbar}, \\
\pi_{n}^{(N)} & =\frac{1}{\hbar^{n} n !} \pi_{0} \bar{a}^{n} a^{n}=\frac{1}{\hbar^{n} n !} \bar{a}^{n} *_{N} \pi_{0}^{(N)} *_{N} a^{n}, \\
E_{n} & =n \hbar \omega .
\end{aligned}
$$

Note that the spectrum obtained in Eq. (5.12) does not include the zero-point energy. The projector onto the ground state $\pi_{0}^{(N)}$ satisfies

$$
a *_{N} \pi_{0}^{(N)}=0 .
$$

The spectral decomposition of the Hamilton function, Eq. (4.4), is in this case

$$
H=\sum_{n=0}^{\infty} n \hbar \omega\left(\frac{1}{\hbar^{n} n !} e^{-a \bar{a} / \hbar} \bar{a}^{n} a^{n}\right)=\omega a \bar{a} .
$$

The Hamilton function is of course a classical quantity; the factor $\hbar$ in the spectrum comes from the deformation parameter in the star product. 
We now consider the Moyal quantization scheme. If we write Eq. (3.6) in terms of holomorphic coordinates, we obtain

$$
f *_{M} g=f e^{\frac{\hbar}{2}\left(\check{\partial}_{a} \vec{\partial}_{\bar{a}}-\overleftarrow{\partial}_{\bar{a}} \vec{\partial}_{a}\right)} g
$$

Here we have

$$
a *_{M} \bar{a}=a \bar{a}+\frac{\hbar}{2}, \quad \bar{a} *_{M} a=a \bar{a}-\frac{\hbar}{2},
$$

and again

$$
[a, \bar{a}]_{*_{M}}=\hbar \text {. }
$$

The value of the commutator of two phase space variables is fixed by property (3) of the star product, and cannot change when one goes to a $c$-equivalent star product. The Moyal star product is $c$-equivalent to the normal star product with the transition operator

$$
T=e^{-\frac{\hbar}{2} \vec{\partial}_{a} \vec{\partial}_{\bar{a}}} .
$$

We can use this operator to transform the normal product version of the $*$-genvalue equation, Eq. (4.3), into the corresponding Moyal product version according to Eq. (3.9). The result is

$$
H *_{M} \pi_{n}^{(M)}=\omega\left(\bar{a} *_{M} a+\frac{\hbar}{2}\right) *_{M} \pi_{n}^{(M)}=\hbar \omega\left(n+\frac{1}{2}\right) \pi_{n}^{(M)},
$$

with

$$
\begin{aligned}
& \pi_{0}^{(M)}=T \pi_{0}^{(N)}=2 e^{-2 a \bar{a} / \hbar}, \\
& \pi_{n}^{(M)}=T \pi_{n}^{(N)}=\frac{1}{\hbar^{n} n !} \bar{a}^{n} *_{M} \pi_{0}^{(M)} *_{M} a^{n} .
\end{aligned}
$$

The projector onto the ground state $\pi_{0}^{(M)}$ satisfies

$$
a *_{M} \pi_{0}^{(M)}=0 .
$$

We now have for the spectrum

$$
E_{n}=\left(n+\frac{1}{2}\right) \hbar \omega
$$

which is the textbook result. We conclude that for this problem the Moyal quantization scheme is the correct one.

The use of the Moyal product in Eq. (4.6) for the star exponential of the harmonic oscillator leads to the following differential equation:

$$
i \hbar \frac{d}{d t} \operatorname{Exp}_{M}(H t)=\left(H-\frac{(\hbar \omega)^{2}}{4} \partial_{H}-\frac{(\hbar \omega)^{2}}{4} H \partial_{H}^{2}\right) \operatorname{Exp}_{M}(H t)
$$

The solution is

$$
\operatorname{Exp}_{M}(H t)=\frac{1}{\cos \frac{\omega t}{2}} \exp \left[\left(\frac{2 H}{i \hbar \omega}\right) \tan \frac{\omega t}{2}\right]
$$

This expression can be brought into the form of the Fourier-Dirichlet expansion of Eq. (4.8) by using the generating function for the Laguerre polynomials:27

$$
\frac{1}{1+s} \exp \left[\frac{z s}{1+s}\right]=\sum_{n=0}^{\infty} s^{n}(-1)^{n} L_{n}(z),
$$

with $s=e^{-i \omega t}$. The projectors then become

$$
\pi_{n}^{(M)}=2(-1)^{n} e^{-2 H / \hbar \omega} L_{n}\left(\frac{4 H}{\hbar \omega}\right)
$$

which is equivalent to the expression already found in Eq. (5.21), as shown in the Appendix. 


\section{Conventional Quantization}

One usually finds the observables characterizing some quantum mechanical system by starting from the corresponding classical system, and then, either by guessing or by using some more or less systematic method, finding the corresponding representations of the classical quantities in the quantum system. The guiding principle is the correspondence principle: the quantum mechanical relations are supposed to reduce somehow to the classical relations in an appropriate limit. Early attempts to systematize this procedure involved finding an assignment rule $\Theta$ that associates to each phase space function $f$ a linear operator in Hilbert space $\hat{f}=\Theta(f)$ in such a way that in the limit $\hbar \rightarrow 0$, the quantum mechanical equations of motion go over to the classical equations. Such an assignment cannot be unique, because even though an operator that is a function of the basic operators $\hat{Q}$ and $\hat{P}$ reduces to a unique phase space function in the limit $\hbar \rightarrow 0$, there are many ways to assign an operator to a given phase space function, due to the different orderings of the operators $\hat{Q}$ and $\hat{P}$ that all reduce to the original phase space function. Different ordering procedures correspond to different quantization schemes. It turns out that there is no quantization scheme for systems with observables that depend on the coordinates or the momenta to a higher power than quadratic which leads to a correspondence between the quantum mechanical and the classical equations of motion, and which_imultaneously strictly maintains the Dirac-von Neumann requirement that $(1 / i \hbar)[\hat{f}, \hat{g}] \leftrightarrow\{f, g\} .9$ Only within the framework of deformation quantization does the correspondence principle acquire a precise meaning.

A general scheme for associating phase space functions and Hilbert space operators, which includes all of the usual orderings, is given as follows.28 The operator $\Theta_{\lambda}(f)$ corresponding to a given phase space function $f$ is

$$
\Theta_{\lambda}(f)=\int \tilde{f}(\xi, \eta) e^{-i(\xi \hat{Q}+\eta \hat{P})} e^{\lambda(\xi, \eta)} d \xi d \eta,
$$

where $\tilde{f}$ is the Fourier transform of $f$, and $(\hat{Q}, \hat{P})$ are the Schrödinger operators that correspond to the phase-space variables $(q, p) ; \lambda(\xi, \eta)$ is a quadratic form:

$$
\lambda(\xi, \eta)=\frac{\hbar}{4}\left(\alpha \eta^{2}+\beta \xi^{2}+2 i \gamma \xi \eta\right) .
$$

Different choices for the constants $(\alpha, \beta, \gamma)$ yield different operator ordering schemes. The choice $\alpha=\beta=0$ is convenient when using $(q, p)$ coordinates; $\gamma=1$ corresponds to antistandard ordering

$$
q p \mapsto \hat{P} \hat{Q},
$$

$\gamma=-1$ to standard ordering

$$
q p \mapsto \hat{Q} \hat{P}
$$

and $\gamma=0$ to the totally symmetric Weyl ordering

$$
q p \mapsto \frac{1}{2}(\hat{Q} \hat{P}+\hat{P} \hat{Q}) .
$$

In holomorphic coordinates it is convenient to take $\gamma=0$, and $\beta=-\alpha$. Then $\alpha=-1$ corresponds to antinormal ordering

$$
a \bar{a} \mapsto \hat{a} \hat{a}^{\dagger},
$$

$\alpha=1$ to normal ordering

$$
a \bar{a} \mapsto \hat{a}^{\dagger} \hat{a},
$$


and $\alpha=0$ to Weyl ordering

$$
a \bar{a} \mapsto \frac{1}{2}\left(\hat{a} \hat{a}^{\dagger}+\hat{a}^{\dagger} \hat{a}\right)
$$

The inverse procedure of finding the phase space function that corresponds to a given operator $\hat{f}$ is, for the special case of Weyl ordering, given by

$$
f(q, p)=\int\left\langle q+\frac{1}{2} \xi|\hat{f}| q-\frac{1}{2} \xi\right\rangle e^{-i \xi p / \hbar} d \xi .
$$

When using holonomic coordinates it is convenient to work with the coherent states 29

$$
\hat{a}|a\rangle=a|a\rangle, \quad\langle\bar{a}| \hat{a}^{\dagger}=\langle\bar{a}| \bar{a} .
$$

These states are related to the energy eigenstates of the harmonic oscillator

$$
|n\rangle=\frac{1}{\sqrt{n !}} \hat{a}^{\dagger}|0\rangle
$$

by

$$
|a\rangle=e^{-\frac{1}{2} a \bar{a} / \hbar} \sum_{n=0}^{\infty} \frac{a^{n}}{\sqrt{n !}}|n\rangle, \quad\langle\bar{a}|=e^{-\frac{1}{2} a \bar{a} / \hbar} \sum_{n=0}^{\infty} \frac{\bar{a}^{n}}{\sqrt{n !}}\langle n| .
$$

In normal ordering we obtain the phase space function $f(a, \bar{a})$ corresponding to the operator $\hat{f}$ by just taking the matrix element between coherent states:

$$
f(a, \bar{a})=\left\langle\bar{a}\left|f\left(\hat{a}, \hat{a}^{\dagger}\right)\right| a\right\rangle .
$$

\section{Quantization, Star Products and Path Integrals}

The relation between operator algebras and star products is given by

$$
\Theta(f) \Theta(g)=\Theta(f * g),
$$

where $\Theta$ is a linear assignment of the kind discussed in Sec. 6. Different assignments, which correspond to different operator orderings, correspond to $c$-equivalent star products. This important relation, which was already known to Groenewold, 19 will be proved in the Appendix. It tells us that the quantum mechanical algebra of observables is a representation of the star product algebra. Because in the algebraic approach to quantum theory all the information concerning the quantum system may be extracted from the algebra of observables, 13 specifying the star product completely determines the quantum system. In particular, if the star product is Hermitean, the operator algebra is a $C^{*}$-algebra. In recent work 30 methods have been developed for constructing explicit Hilbert space representations of the deformed star product algebra: here the algebra of observables is the primary object and the representing Hilbert spaces are subordinate. In this sense deformation quantization is not just an alternative approach to quantum theory: it may be considered as a specification of the basic quantum structure.

In the conventional approach the time-development of the system is characterized by the appropriate matrix element of the time-development operator, namely the Feynman kernel:

$$
K\left(q_{2}, t ; q_{1}, 0\right)=\left\langle q_{2}\left|e^{-i \hat{H} t / \hbar}\right| q_{1}\right\rangle,
$$

where $\hat{H}$ is the Hamilton operator. By substituting a complete set of energy eigenstates, we obtain an expression resembling the Fourier-Dirichlet expansion of Sec. 4:

$$
K\left(q_{2}, t ; q_{1}, 0\right)=\sum_{n=0}^{\infty}\left\langle q_{2} \mid n\right\rangle\left\langle n \mid q_{1}\right\rangle e^{-i E_{n} t / \hbar} .
$$


For the harmonic oscillator we may insert the known eigenfunctions and eigenvalues to obtain the following expression for $K\left(q_{2}, t ; q_{1}, 0\right)$ :

$$
\begin{array}{r}
\frac{1}{2^{n} n !} \sqrt{\frac{m \omega}{\pi \hbar}} e^{-\frac{m \omega}{\hbar}\left(q_{1}^{2}+q_{2}^{2}\right)} \sum_{n=0}^{\infty} e^{-i\left(n+\frac{1}{2}\right) \omega t} H_{n}\left(\sqrt{\frac{m \omega}{\hbar}} q_{1}\right) H_{n}\left(\sqrt{\frac{m \omega}{\hbar}} q_{2}\right) \\
=\sqrt{\frac{m \omega}{2 \pi i \hbar \sin \omega t}} \exp \left[\frac{i m \omega}{2 \hbar \sin \omega t}\left(\left(q_{1}^{2}+q_{2}^{2}\right) \cos \omega t-2 q_{1} q_{2}\right)\right],
\end{array}
$$

where we have used the following expansion formula involving the Hermite polynomials 27

$$
\frac{1}{\sqrt{1-s^{2}}} \exp \left[\frac{2 x y s-s^{2}\left(x^{2}+y^{2}\right)}{1-s^{2}}\right]=\sum_{n=0}^{\infty} \frac{s^{n}}{2^{n} n !} H_{n}(x) H_{n}(y) .
$$

In order to relate this to the phase space functions for the harmonic oscillator discussed in Sec. 4, we apply the Fourier transform of Eq. (6.9) to both sides of Eq. (7.4). We use the following relation between the Hermite and Laguerre polynomials:

$$
\int d x\left[H_{n}(x-a) H_{n}(x+a) e^{-x^{2}}\right] e^{-2 i b x}=2^{n} \sqrt{\pi} n ! e^{-b^{2}} L_{n}\left(2\left(a^{2}+b^{2}\right)\right),
$$

and find

$$
2(-1)^{n} \sum_{n=0}^{\infty} e^{-i\left(n+\frac{1}{2}\right) \omega t} e^{-\frac{2 H}{\hbar \omega}} L_{n}\left(\frac{4 H}{\hbar \omega}\right)=\frac{1}{\cos \frac{\omega t}{2}} \exp \left[\frac{2 H}{i \hbar \omega} \tan \frac{\omega t}{2}\right] .
$$

The right-hand side of Eq. (7.7) is the expression for the Moyal star exponential for the harmonic oscillator as given in Eq. (5.25). According to the Fourier-Dirichlet expansion formula (4.8) the left-hand side gives the expressions (5.27) for the projectors. For holomorphic coordinates the calculation is even easier:

$$
\pi_{n}^{(N)}(a, \bar{a})=\frac{1}{\hbar^{n}}\langle\bar{a} \mid n\rangle\langle n \mid a\rangle=\frac{1}{\hbar^{n} n !}(\bar{a} a)^{n} e^{-\bar{a} a / \hbar},
$$

in agreement with Eq. (5.11) for the normal star product projectors.

We see that the star exponential $\operatorname{Exp}(H t)$ and the projectors $\pi_{n}$ are the phase space representations of the time-evolution operator $e^{-i \hat{H} t / \hbar}$ and the projection operators $\hat{\rho}_{n}=|n\rangle\langle n|$, respectively. Weyl-ordering corresponds to the use of the Moyal star product for quantization and normal ordering to the use of the normal star product. In the density matrix formalism we say that the projection operator is that of a pure state, which is characterized by the property of being idempotent: $\hat{\rho}_{n}^{2}=\hat{\rho}_{n}$ (compare Eq. (4.2)). The integral of the projector over the momentum gives the probability distribution in position space:

$$
\begin{aligned}
\frac{1}{2 \pi \hbar} \int \pi_{n}^{(M)}(q, p) d p & =\frac{1}{2 \pi \hbar} \int\langle q+\xi / 2 \mid n\rangle\langle n \mid q-\xi / 2\rangle e^{-i \xi p / \hbar} d \xi d p \\
& =\langle q \mid n\rangle\langle n \mid q\rangle=\left|\psi_{n}(q)\right|^{2}
\end{aligned}
$$

and the integral over the position gives the probability distribution in momentum space:

$$
\frac{1}{2 \pi \hbar} \int \pi_{n}^{(M)}(q, p) d q=\langle p \mid n\rangle\langle n \mid p\rangle=\left|\tilde{\psi}_{n}(p)\right|^{2} .
$$

The normalization is

$$
\frac{1}{2 \pi \hbar} \int \pi_{n}^{(M)}(q, p) d q d p=1
$$


which is the same as Eq. (4.1). Applying these relations to the ground state projector of the harmonic oscillator, Eq. (5.20) shows that this is a minimum-uncertainty state. In the classical limit $\hbar \rightarrow 0$, it goes to a Dirac $\delta$-function. The expectation value of the Hamiltonian operator is

$$
\frac{1}{2 \pi \hbar} \int\left(H *_{M} \pi_{n}^{(M)}\right)(q, p) d q d p=\int\left\langle q\left|\hat{H} \hat{\rho}_{n}\right| q\right\rangle d q=\operatorname{Tr}\left(\hat{H} \hat{\rho}_{n}\right),
$$

which should be compared to Eq. (4.5).

The relation (7.1) agrees nicely with the interpretation of the time evolution of the quantum mechanical system suggested by Feynman. In this view the time evolution for a finite time interval is the result of successive steps corresponding to time intervals $\Delta t=t / N$. In the operator formalism the finite time evolution operator is thus the product of the short-time evolution operators

$$
\left\langle q_{i+1}, t_{i+1} \mid q_{i}, t_{i}\right\rangle=\left\langle q_{i+1}\left|e^{-i \hat{H} \Delta t / \hbar}\right| q_{i}\right\rangle .
$$

By Eq. (7.1) this procedure corresponds to forming the star product of the associated phase space functions $\operatorname{Exp}(H \Delta t)$. In this way the star exponential which results for the finite time evolution is the Fourier transform of the Feynman kernel in the sense of Eq. 6.9). Feynman's procedure yields the path integral expression for the kernel

$$
K\left(q_{2}, t ; q_{1}, 0\right)=\int D q(t) e^{i S[q] / \hbar},
$$

where $S[q]$ is the classical action functional and the notation $D q(t)$ indicates an integration over all paths with the fixed endpoints $q_{1}$ and $q_{2}$. For the harmonic oscillator the semi-classical approximation is exact, and hence the path integral can be evaluated by inserting the classical solution into the action functional. The result of this calculation agrees with the right-hand side of Eq. (7.4. 31 Hence, there is also a direct relationship between the path integral and the star exponential. This relationship has been verified directly in the general case by Sharan 32 for the coordinate representation, and by Dito 33 for the holomorphic representation.

\section{Quantum Field Theory}

A real scalar field is given in terms of the coefficients $a(\mathbf{k}), \bar{a}(\mathbf{k})$ by

$$
\phi(x)=\int \frac{d^{3} k}{(2 \pi)^{\frac{3}{2}} \sqrt{2 \omega_{\mathbf{k}}}}\left[a(\mathbf{k}) e^{-i k x}+\bar{a}(\mathbf{k}) e^{i k x}\right],
$$

where $\hbar \omega_{\mathbf{k}}=\sqrt{\hbar^{2} k^{2}+m^{2}}$ is the energy of a single quantum of the field. The corresponding quantum field operator is

$$
\Phi(x)=\int \frac{d^{3} k}{(2 \pi)^{\frac{3}{2}} \sqrt{2 \omega_{\mathbf{k}}}}\left[\hat{a}(\mathbf{k}) e^{-i k x}+\hat{a}^{\dagger}(\mathbf{k}) e^{i k x}\right],
$$

where $\hat{a}(\mathbf{k}), \hat{a}^{\dagger}(\mathbf{k})$ are the annihilation and creation operators for a quantum of the field with momentum $\hbar \mathbf{k}$. The Hamiltonian is

$$
H=\int d^{3} k \hbar \omega_{\mathbf{k}} \hat{a}^{\dagger}(\mathbf{k}) \hat{a}(\mathbf{k}) .
$$

$N(\mathbf{k})=\hat{a}^{\dagger}(\mathbf{k}) \hat{a}(\mathbf{k})$ is interpreted as the number operator, and Eq. (8.3) is then just the generalization of Eq. (5.12), the expression for the energy of the harmonic oscillator in the normal ordering scheme, for an infinite number of degrees of freedom. Had we chosen the Weyl ordering scheme, 
we would have been lead, by the generalization of Eq. (5.23), to an infinite vacuum energy. Hence requiring the vacuum energy to vanish implies the choice of the normal ordering scheme in free field theory. In the framework of deformation quantization this requirement leads to the choice of the normal star product for treating free scalar fields, as pointed out by Dito: 33 only for this choice is the star product well-defined.

Currently, in realistic physical field theories involving interacting relativistic fields we are limited to perturbative calculations. The objects of interest are products of the fields. The analog of the Moyal product of Eq. (3.5) for systems with an infinite number of degrees of freedom is

$$
\begin{aligned}
& \phi\left(x_{1}\right) * \phi\left(x_{2}\right) * \cdots * \phi\left(x_{n}\right) \\
& =\exp \left[\frac{1}{2} \sum_{i<j} \int d^{4} x d^{4} y \frac{\delta}{\delta \phi_{i}(x)} \Delta(x-y) \frac{\delta}{\delta \phi_{j}(y)}\right] \phi_{1}\left(x_{1}\right),\left.\ldots \phi_{n}\left(x_{n}\right)\right|_{\phi_{i}=\phi},
\end{aligned}
$$

where the expressions $\delta / \delta \phi(x)$ indicate functional derivatives. Here we have used the antisymmetric Schwinger function

$$
\Delta(x-y)=[\Phi(x), \Phi(y)] .
$$

The Schwinger function is uniquely determined by relativistic invariance and causality from the equal-time commutator

$$
\left.[\Phi(x), \dot{\Phi}(y)]\right|_{x^{0}=y^{0}}=i \hbar \delta^{(3)}(\mathbf{x}-\mathbf{y}),
$$

which is the characterization of the canonical structure in the field theoretic framework.

The Moyal product is, however, not the suitable star product to use in this context. In relativistic quantum field theory it is necessary to incorporate causality in the form advocated by Feynman: positive frequencies propagate forward in time, whereas negative frequencies propagate backwards in time. This property is achieved by using the Feynman propagator:

$$
\Delta_{F}(x)=\left\{\begin{aligned}
\Delta^{+}(x) & \text { for } x^{0}>0 \\
-\Delta^{-}(x) & \text { for } x^{0}<0
\end{aligned}\right.
$$

where $\Delta^{+}(x), \Delta^{-}(x)$ are the propagators for the positive and negative frequency components of the field, respectively. In operator language

$$
\Delta_{F}(x-y)=\mathcal{T}(\Phi(x) \Phi(y))-\mathcal{N}(\Phi(x) \Phi(y)),
$$

where $\mathcal{T}$ indicates the time-ordered product of the fields and $\mathcal{N}$ the normal-ordered product. Because the second term in Eq. (8.8) is a normal ordered product with vanishing vacuum expectation value, the Feynman propagator may be simply characterized as the vacuum expectation value of the time-ordered product of the fields. The antisymmetric part of the positive frequency propagator is the Schwinger function:

$$
\Delta^{+}(x)-\Delta^{+}(-x)=\Delta^{+}(x)+\Delta^{-}(x)=\Delta(x) .
$$

The fact that going over to a $c$-equivalent product leaves the antisymmetric part of the differential operator in the exponent of Eq. (8.4) invariant suggests that the use of the positive frequency propagator instead of the Schwinger function merely involves the passage to a $c$-equivalent star product. This is indeed easy to verify. The time-ordered product of the operators is obtained by replacing the Schwinger function $\Delta(x-y)$ in Eq. (8.4) by the c-equivalent positive frequency propagator $\Delta^{+}(x-y)$, restricting the time integration to $x^{0}>y^{0}$, as in Eq. (8.7), and symmetrizing the integral in the variables $x$ and $y$, which brings in the jnegative frequency propagator $\Delta^{-}(x-y)$ 
for times $x^{0}<y^{0}$. Then Eq. (8.4) becomes Wick's theorem, which is the basic tool of relativistic perturbation theory. In operator language 34

$$
\mathcal{T}\left(\Phi\left(x_{1}\right), \ldots \Phi\left(x_{n}\right)\right)=\exp \left[\frac{1}{2} \int d^{4} x d^{4} y \frac{\delta}{\delta \Phi(x)} \Delta_{F}(x-y) \frac{\delta}{\delta \Phi(y)}\right] \mathcal{N}\left(\Phi\left(x_{1}\right), \ldots \Phi\left(x_{n}\right)\right)
$$

The relation between relativistic perturbatip theory and deformation quantization has recently been discussed by Dütsch and Fredenhagen.14

Another interesting relation between deformation quantization and quantum field theory has been uncovered by studies of the Poisson-Sigma model 35 This model involves a set of scalar fields $X^{i}$ which map a two-dimensional manifold $\Sigma_{2}$ onto a Poisson space $M$, as well as generalized gauge fields $A_{i}$, which are one-forms on $\Sigma_{2}$ mapping to one-forms on $M$. The action is given by

$$
S_{P S}=\int_{\Sigma_{2}}\left(A_{i} d X^{i}+\alpha^{i j} A_{i} A_{j}\right)
$$

where $\alpha^{i j}$ is the Poisson structure of $M$. The remarkable formula found by Cattaneo and Felder 36 is

$$
(f * g)(x)=\int D X D A f(X(1)) g(X(2)) e^{i S_{P S} / \hbar},
$$

where $f, g$ are functions on $M, *$ is Kontsevich's star product 15 and the functional integration is over all fields $X$ that satisfy the boundary condition $X(\infty)=x$. Here $\Sigma_{2}$ is taken to be a disc in $\mathbb{R}^{2} ; 1,2$, and $\infty$ are three points on its circumference. By expanding the functional integral in Eq. (8.12) according to the usual rules of perturbation theory, one finds that the coefficients of the powers of $\hbar$ reproduce the graphs and weights that characterize Kontsevich's star product. For the case in which the Poisson tensor is invertible, we can perform the Gaussian integration in Eq. (8.12) involving the fields $A_{i}$. The result is

$$
(f * g)(x)=\int D X f(X(1)) g(X(2)) \exp \left[\frac{i}{\hbar} \int \Omega_{i j} d X^{i} d X^{j}\right] .
$$

Equation (8.13) is formally similar to Eq. (3.14) for the Moyal product, to which the Kontsevich product reduces in the symplectic case. Here $\Omega_{i j}=\left(\alpha^{i j}\right)^{-1}$ is the symplectic 2 -form, and $\int \Omega_{i j} d X^{i} d X^{j}$ is the symplectic volume of the manifold $M$. To make this relationship exact one must integrate out the gauge degrees of freedom in the functional integral in Eq. (8.13). Since the Poisson-sigma model represents a topological field theory there would remain only a finite-

dimensional integral, which would coincide with the integral in Eq. (3.17). For details of this procedure see Ref. (37).

Another important application of deformation theory in the field theoretic context involves the correct treatment of unphysical degrees of freedom in gauge theories. Currently it is believed that deformation theory is the best method for dealing with these systems. 38 There are also attempts to use the methods of deformation quantization to treat problems in string theory 39 and quantum gravity. 40

\section{Acknowledgements}

We wish to thank Dipl. Cand. Stefan Jansen from Dortmund, who helped us with some of the calculations which appear in this paper. P. H. acknowledges a Stipendium for Graduate Studies from the Land Nordrhein-Westfalen. A. C. H. wishes to thank the organizers of the Third International Conference on Geometry, Integrability and Quantization, held in Varna, Bulgaria from June 14-23, 2001, for the opportunity to present a preliminary version of this material. 


\section{A Appendix: Calculational Techniques}

Questions related to deformation quantization have attracted the interest of many prominent mathematicians, and there is an extensive mathematical literature on the subject. We nevertheless wish to emphasize that physics students learning quantum mechanics should become proficient in performing calculations in the same style that they employ in their other subjects, for example in classical electrodynamics. That is, they should fearlessly interchange the order of summations and integrations, naively manipulate delta functions, etc. Physicists have usually achieved their results by quick and ready calculations, which were only later justified by their mathematical colleagues. In any case, the ultimate test for physical theories is the comparison to experiment.

To encourage students on this path, we present in this Appendix a few typical calculations of this kind which can be used to motivate some of the relations in the main text. After working through these examples, students should be able to cope with the other results discussed.

We shall first look at the important Eq. (7.1) which relates star products and operator products. Although the relation holds for any specific ordering and its corresponding star product, we shall perform the calculation using Weyl ordering. The product of the two operators $\Theta(f)$ and $\Theta(g)$, which represent the phase space functions $f$ and $g$, respectively, is

$$
\begin{aligned}
\Theta(f) \Theta(g)= & \int d \xi_{1} d \eta_{1} d \xi_{2} d \eta_{2} \tilde{f}\left(\xi_{1}, \eta_{1}\right) \tilde{g}\left(\xi_{2}, \eta_{2}\right) \\
& \times \exp \left[-i\left(\xi_{1} \hat{Q}+\eta_{1} \hat{P}\right)\right] \exp \left[-i\left(\xi_{2} \hat{Q}+\eta_{2} \hat{P}\right)\right] \\
= & \int d \xi_{1} d \eta_{1} d \xi_{2} d \eta_{2} \tilde{f}\left(\xi_{1}, \eta_{1}\right) \tilde{g}\left(\xi_{2}, \eta_{2}\right) \\
& \times \exp \left[-i\left(\left(\xi_{1}+\xi_{2}\right) \hat{Q}+\left(\eta_{1}+\eta_{2}\right) \hat{P}\right)\right] \exp \left[\frac{-i \hbar}{2}\left(\xi_{1} \eta_{2}-\eta_{1} \xi_{2}\right)\right],
\end{aligned}
$$

where we have used the truncated Campbell-Baker-Hausdorff formula:

$$
e^{A} e^{B}=e^{(A+B)} e^{\frac{1}{2}[A, B]} .
$$

We expand the last exponential in Eq. A.1), make the substitution of variables $\xi=\xi_{1}+\xi_{2}$, $\eta=\eta_{1}+\eta_{2}$, and obtain

$$
\begin{aligned}
& \Theta(f) \Theta(g)=\int d \xi d \eta e^{-i(\xi \hat{Q}+\eta \hat{P})} \\
& \quad \times \int d \xi_{1} d \eta_{1} \sum_{m, n=0}^{\infty} \frac{(-1)^{m}}{m ! n !}\left(\frac{i \hbar}{2}\right)^{m+n} \xi_{1}^{m} \eta_{1}^{n} \tilde{f}\left(\xi_{1}, \eta_{1}\right)\left(\xi-\xi_{1}\right)^{n}\left(\eta-\eta_{1}\right)^{m} \tilde{g}\left(\xi-\xi_{1}, \eta-\eta_{1}\right) .
\end{aligned}
$$

The expression on the second line of Eq. (A.3) is by the Fourier convolution theorem just the Fourier transform of the expression for the Moyal product in Eq. (3.7). Hence

$$
\Theta(f) \Theta(g)=\int d \xi d \eta\left(\widetilde{f *_{M}} g\right) e^{-i(\xi \hat{Q}+\eta \hat{P})}=\Theta\left(f *_{M} g\right) .
$$

To get the representation for the Moyal product of Eq. (3.14), we use again the Fourier convo- 
lution theorem and write the Fourier transforms of the functions $f$ and $g$ explicitly:

$$
\begin{aligned}
f *_{M} g & =\frac{1}{4 \pi^{2}} \int d \tau d \sigma d \xi d \eta d q_{1} d p_{1} d q_{2} d p_{2} e^{i \sigma q} e^{i \tau p} \\
& \times e^{\frac{i \hbar}{2}(\eta(\sigma-\xi)-\xi(\tau-\eta))} e^{-i \xi q_{1}-i \eta p_{1}} f\left(q_{1}, p_{1}\right) e^{-i(\sigma-\xi) q_{2}-i(\tau-\eta) p_{2}} g\left(q_{2}, p_{2}\right) \\
& =\frac{1}{4 \pi^{2}} \int d \tau d \sigma d \xi d \eta d q_{1} d p_{1} d q_{2} d p_{2} f\left(q_{1}, p_{1}\right) g\left(q_{2}, p_{2}\right) \\
& \times \exp \left[i \sigma\left(q+\frac{\hbar}{2} \eta-q_{2}\right)+i \tau\left(p-\frac{\hbar}{2} \xi-p_{2}\right)-i \xi q_{1}-i \eta p_{1}+i \xi q_{2}+i \eta p_{2}\right] \\
& =\frac{1}{4 \pi^{2}} \int d \xi d \eta d q_{1} d p_{1} d q_{2} d p_{2} f\left(q_{1}, p_{1}\right) g\left(q_{2}, p_{2}\right) \\
& \times \delta\left(-q-\frac{\hbar}{2} \eta+q_{2}\right) \delta\left(-p+\frac{\hbar}{2} \xi+p_{2}\right) \exp \left[-i \xi q_{1}-i \eta p_{1}+i \xi q_{2}+i \eta p_{2}\right] .
\end{aligned}
$$

Now rescale the delta functions according to $\delta\left(-q-\frac{\hbar}{2} \eta+q_{2}\right)=\left(\frac{2}{\hbar}\right) \delta\left(\eta+\frac{2}{\hbar} q-\frac{2}{\hbar} q_{2}\right)$, and similarly for the second delta function, and perform the $\xi$ and $\eta$ integrations. The result is Eq. (3.14).

The result in Eq. (5.27) expressing the projectors of the harmonic oscillator in the Moyal scheme in terms of the Laguerre polynomials may be obtained directly by calculating the expressions $\pi_{n}^{(M)}=T \pi_{n}^{(N)}$ given in Eq. (5.21). These expressions can be written as

$$
\begin{aligned}
T \pi_{n}^{(N)} & =\exp \left(-\frac{\hbar}{2} \partial_{a} \partial_{\bar{a}}\right) \frac{1}{\hbar^{n} n !} \bar{a}^{n} a^{n} e^{-a \bar{a} / \hbar} \\
& =\frac{1}{\hbar^{n} n !} \bar{a}^{n} a^{n} \exp \left(-\frac{\hbar}{2}\left(\overleftarrow{\partial}_{a} \overleftarrow{\partial}_{\bar{a}}+\overleftarrow{\partial}_{a} \vec{\partial}_{\bar{a}}+\overleftarrow{\partial}_{\bar{a}} \vec{\partial}_{a}+\vec{\partial}_{a} \vec{\partial}_{\bar{a}}\right)\right) e^{-a \bar{a} / \hbar}
\end{aligned}
$$

In principle, one has to include the commutator in the Campbell-Baker-Hausdorff formula when factorizing exponential terms, but in this case the commutator vanishes. Hence, we can factor out the last term in the exponent and apply Eq. (5.20) for $\pi_{0}^{(N)}$ to get

$$
\begin{aligned}
& \frac{2}{\hbar^{n} n !} \bar{a}^{n} a^{n} \exp \left(-\frac{\hbar}{2}\left(\overleftarrow{\partial}_{a} \overleftarrow{\partial}_{\bar{a}}+\overleftarrow{\partial}_{a} \vec{\partial}_{\bar{a}}+\overleftarrow{\partial}_{\bar{a}} \vec{\partial}_{a}\right)\right) e^{-2 a \bar{a} / \hbar} \\
= & \frac{2}{\hbar^{n} n !} \bar{a}^{n} a^{n} \exp \left(-\frac{\hbar}{2}\left(\overleftarrow{\partial}_{a} \overleftarrow{\partial}_{\bar{a}}+\overleftarrow{\partial}_{a} \vec{\partial}_{\bar{a}}\right)\right) \exp \left(-\frac{\hbar}{2}\left(\overleftarrow{\partial}_{\bar{a}} \vec{\partial}_{a}\right)\right) e^{-2 a \bar{a} / \hbar} \\
= & \frac{2}{\hbar^{n} n !} \bar{a}^{n} a^{n} \exp \left(-\frac{\hbar}{2}\left(\overleftarrow{\partial}_{a} \overleftarrow{\partial}_{\bar{a}}+\overleftarrow{\partial}_{a} \vec{\partial}_{\bar{a}}\right)\right) \exp \left(\overleftarrow{\partial}_{\bar{a}} \bar{a}\right) e^{-2 a \bar{a} / \hbar} .
\end{aligned}
$$

Because the commutator $\left[-\frac{\hbar}{2}\left(\overleftarrow{\partial}_{a} \overleftarrow{\partial}_{\bar{a}}+\overleftarrow{\partial}_{a} \vec{\partial}_{\bar{a}}\right), \overleftarrow{\partial}_{\bar{a}} \bar{a}\right]$ vanishes, we can exchange the order of the two exponentials in the last equation and then carry out the operations indicated by the first exponential:

$$
\begin{aligned}
& \frac{2}{\hbar^{n} n !} \bar{a}^{n} a^{n} \exp \left(\overleftarrow{\partial}_{\bar{a}} \bar{a}\right) \exp \left(-\frac{\hbar}{2}\left(\overleftarrow{\partial}_{a} \overleftarrow{\partial}_{\bar{a}}+\overleftarrow{\partial}_{a} \vec{\partial}_{\bar{a}}\right)\right) e^{-2 a \bar{a} / \hbar} \\
= & \frac{2}{\hbar^{n} n !}(\bar{a}+\bar{a})^{n} a^{n} \exp \left(-\frac{\hbar}{2}\left(\overleftarrow{\partial}_{a} \overleftarrow{\partial}_{\bar{a}}+\overleftarrow{\partial}_{a} \vec{\partial}_{\bar{a}}\right)\right) e^{-2 a \bar{a} / \hbar} \\
= & \frac{2}{\hbar^{n} n !} 2^{n} \bar{a}^{n} a^{n} \exp \left(-\frac{\hbar}{2}\left(\overleftarrow{\partial}_{a} \overleftarrow{\partial}_{\bar{a}}\right)\right) \exp \left(\overleftarrow{\partial}_{a} a\right) e^{-2 a \bar{a} / \hbar}
\end{aligned}
$$

Here we have used the Taylor formula in the form

$$
f(x+a)=e^{a \partial_{x}} f(x) .
$$


The first exponential can now be expanded:

$$
\begin{aligned}
& \frac{2}{\hbar^{n} n !} 2^{n}\left(\sum_{k=0}^{\infty} \frac{1}{k !}\left(-\frac{\hbar}{2}\right)^{k}\left(\partial_{a}^{k} a^{n}\right)\left(\partial_{\bar{a}}^{k} \bar{a}^{n}\right)\right) \exp \left(\overleftarrow{\partial}_{a} a\right) e^{-2 a \bar{a} / \hbar} \\
= & 2\left(\sum_{k=0}^{n} \frac{(-1)^{k}}{k !}\left(\frac{2}{\hbar}\right)^{n-k} \frac{n !}{(n-k) !(n-k) !} a^{n-k} \bar{a}^{n-k}\right) \exp \left(\overleftarrow{\partial}_{a} a\right) e^{-2 a \bar{a} / \hbar} \\
= & (-1)^{n} 2 L_{n}(2 a \bar{a} / \hbar) \exp \left(\overleftarrow{\partial}_{a} a\right) e^{-2 a \bar{a} / \hbar} \\
= & (-1)^{n} 2 L_{n}(4 a \bar{a} / \hbar) e^{-2 a \bar{a} / \hbar}
\end{aligned}
$$

where we used the definition of the Laguerre polynomials:27

$$
L_{n}(x)=\sum_{m=0}^{n}(-1)^{m} \frac{n !}{(n-m) ! m ! m !} x^{m} .
$$

Using similar methods students should now be able to do the following problems.

1. Show that Eq. (3.12) gives the transition operator from the standard star product to the Moyal product, that is, that Eq. (3.11) is satisfied.

2. Repeat the calculation above for the projectors $\pi_{n}^{(M)}$ to obtain the form of the projector $\pi_{0}^{(M)}$ given in Eq. (5.20).

3. Verify that the projectors $\pi_{0}^{(N)}$ and $\pi_{0}^{(M)}$ satisfy the idempotence property of Eq. (4.2). You may find the Fourier form of the Moyal product, Eq. (3.14), convenient for this purpose.

4. Show that the prescriptions for passing from a phase-space function to its corresponding Weyl-ordered operator and back, given in Eqs. (6.1) and (6.9), are really inverse to each other.

5. Establish the relation between the Laguerre and Hermite polynomials given in Eq. (7.6).

6. Perform the path integral indicated in Eq. (7.14 for the harmonic oscillator in order to obtain the expression (7.4) for the Feynman kernel.

7. Perform the Gaussian integration which reduces Eq. (8.12) to Eq. 8.13) when the Poisson tensor is invertible. 


\section{References}

[1] P. A. M. Dirac, The Principles of Quantum Mechanics (Clarendon Press, Oxford, 1930).

[2] J. von Neumann, Mathematische Grundlagen der Quantenmechanik (Springer Verlag, Berlin 1932). Math. Ann. 104, 570-578 (1931).

[3] R. P. Feynman, "Space-time approach to non-relativistic quantum mechanics," Rev. Mod. Phys. 20, 367-387 (1947).

[4] J. J. Sakurai, Modern Quantum Mechanics (Addison Wesley, Reading, Massachusetts 1994).

[5] M. E. Peskin and D. Schroeder, An Introduction to Quantum Field Theory (Perseus Books, Reading, Massachusetts, 1995).

[6] E. P. Wigner, "Quantum corrections for thermodynamic equilibrium," Phys. Rev. 40, 749759 (1932).

[7] H. Weyl, Quantenmechanik und Gruppentheorie (Hirzel Verlag, Leipzig, 1928). Z. Phys. 46, 1-46 (1927).

[8] F. Bayen, M. Flato, C. Fronsdal, A. Lichnerowicz, and D. Sternheimer, "Deformation theory and quantization I, II," Ann. Phys. (NY) 111, 61-110, 111-151 (1978).

[9] G. Dito and D. Sternheimer, "Deformation quantization: genesis, developments and metamorphoses", in Deformation quantization (G. Halbout, ed.), IRMA Lectures in Math. Physics I, 9-54, (Walter de Gruyter, Berlin, 2002). math.QA/0220116 8 (2002).

[10] S. Gutt, "Variations on deformation quantization," presented at the Moshe Flato Conference, Dijon, 1999, math.DG/0003107 (2000).

[11] A. Weinstein, "Deformation quantization," Séminaire Bourbaki, Asterique 789, 389-409 (1995).

[12] C. Zachos, "Deformation quantization: quantum mechanics lives and works in phase space," hep-th/0110114 (2001).

[13] R. Haag, Local Quantum Physics (Springer Verlag, Berlin, 1992).

[14] M. Dütsch and K. Fredenhagen, "Perturbative algebraic field theory and deformation quantization," hep-th/0101079 (2001).

[15] M. Kontsevich, "Deformation quantization of Poisson manifolds," q-alg/9709040 (1997).

[16] J. Marsden and T. Ratiu, Introduction to Mechanics and Symmetry (Texts in App. Math. 17, Springer Verlag, 1994).

[17] I. Vaisman, Geometry of Poisson Manifolds (Birkhäuser Verlag, Basel, 1994).

[18] A. Cannas da Silva and A. Weinstein, Geometric Models for Noncommutative Algebras (Amer. Math. Soc. ISBN 0-8218-0952-0, 1999).

[19] H. J. Groenewold, "On the principles of elementary quantum mechanics," Physica 12, 405-460 (1946).

[20] M. Gerstenhaber, "On the deformation of rings and algebras," Ann. Math. 79, 59-103 (1964).

[21] M. DeWilde and P. B. A. Lecomte, "Existence of star-products and of formal deformations of the Poisson Lie algebra of arbitrary symplectic manifolds," Lett. Math. Phys. 7, 487-496 (1983). 
[22] J. E. Moyal, "Quantum mechanics as a statistical theory," Proc. Cambridge Phil. Soc. 45, 99-124 (1949).

[23] M. Bertelson, M. Cahen, and S. Gutt, "Equivalence of star products," Class. Quantum Gravity 14, A93-A107 (1997).

[24] F. Hansen, "Quantum mechanics in phase space," Rep. Math. Phys. 19, 361-381 (1984).

[25] M. A. Rieffel, "Deformation quantization of Heisenberg manifolds," Comm. Math. Phys. 122, 531-562 (1989).

[26] C. Zachos, "A survey of star product geometry," hep-th/000801002 (2000).

[27] P. M. Morse and H. Feshbach, Methods of Theoretical Physics (McGraw-Hill, New York, 1953).

[28] G. S. Agarwal and E. Wolf, "Calculus for functions of noncommuting operators and general phase-space methods in quantum mechanics I," Phys. Rev. D10, 2161-2186 (1970).

[29] R. J. Glauber, "Coherent and incoherent states of the radiation field," Phys. Rev. 131, 2766-2788 (1963).

[30] M. Bordemann and S. Waldmann "Formal GNS construction and states in deformation quantization," Comm. Math. Phys. 195, 549-583 (1998); M. Bordemann, N. Neumaier, and S. Waldmann, "Homogeneous Fedosov star products on cotangent bundles I: Weyl and standard ordering with differential operator representation," Comm. Math. Phys. 198, 363-396 (1998).

[31] R. P. Feynman and A. R. Hibbs, Quantum Mechanics and Path Integrals (McGraw-Hill, New York, 1965).

[32] P. Sharan, "Star-product representation of path integrals," Phys. Rev. D20, 414-418 (1979).

[33] J. Dito, "Star-product approach to quantum field theory: the free scalar field," Lett. Math. Phys. 20, 125-134 (1990).

[34] H. Leschke, A. C. Hirshfeld, and T. Suzuki, "Canonical perturbation theory for nonlinear systems," Phys. Rev. D18, 2834-2848 (1978).

[35] P. Schaller and T. Strobl, "Poisson structure induced (topological) field theories in two dimensions," Mod. Phys. Lett. A9, 3129-3136 (1994).

[36] A. S. Cattaneo and G. Felder, "A path integral approach to the Kontsevich quantization formula," Comm. Math. Phys. 212, 591-611 (2000).

[37] A. C. Hirshfeld and T. Schwarzweller, "Path integral quantization of the Poisson-Sigma model," Ann. Phys. (Leipzig) 9, 83-101 (2000).

[38] J. Stasheff, "Deformation theory and the Batalin-Vilkovisky master equation," q-alg/9702012 (1997).

[39] H. Garcia-Compean, J. F. Plebanski, M. Przanowski, and F. J. Turrubiates, "Deformation quantization of bosonic strings," J. Phys. A33, 7935-7954 (2000).

[40] F. Antonsen, "Deformation quantization of gravity," Bohr Institute preprint (1997). 